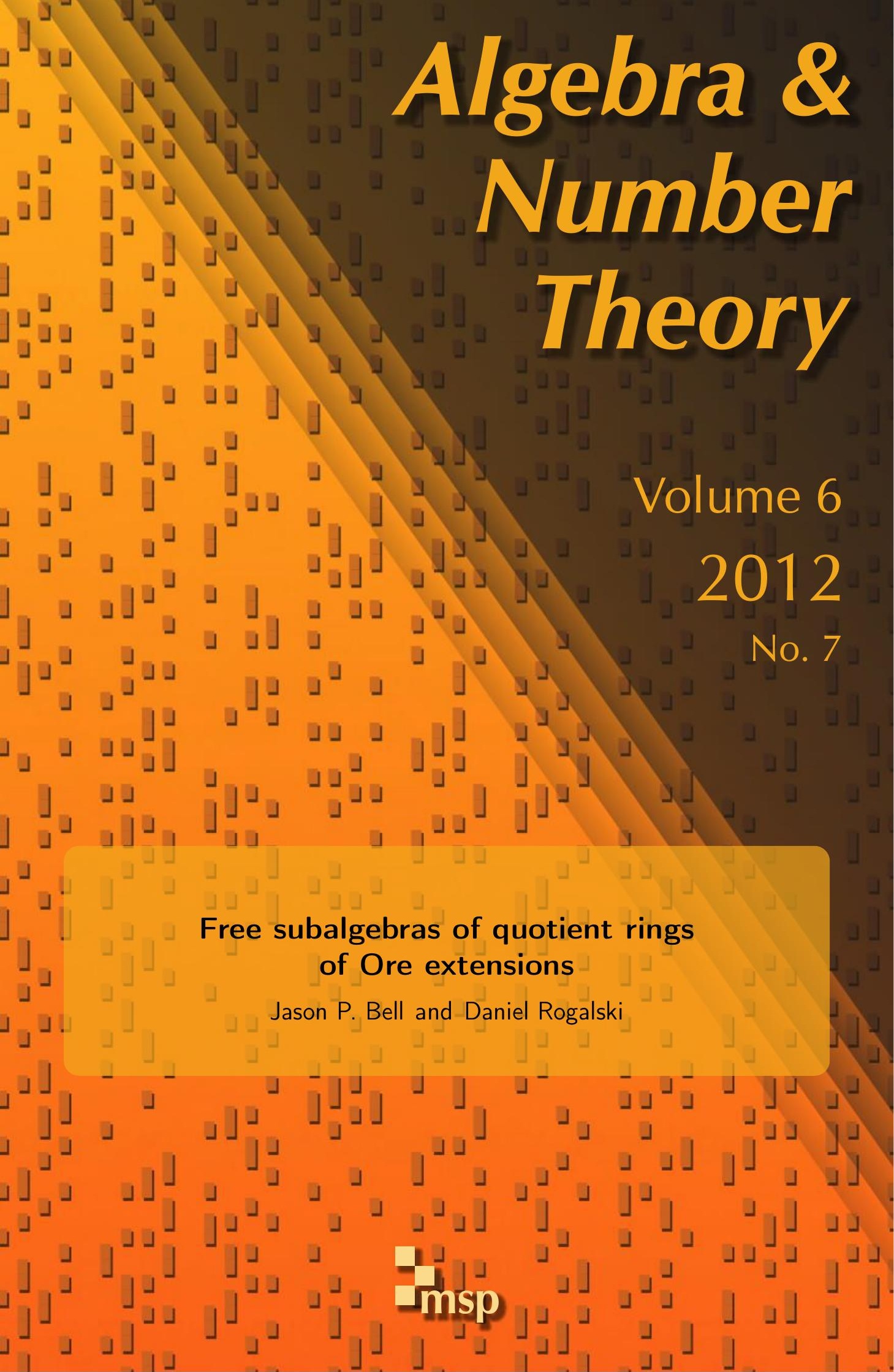




\title{
Free subalgebras of quotient rings of Ore extensions
}

\author{
Jason P. Bell and Daniel Rogalski
}

Let $K$ be a field extension of an uncountable base field $k$, let $\sigma$ be a $k$-automorphism of $K$, and let $\delta$ be a $k$-derivation of $K$. We show that if $D$ is one of $K(x ; \sigma)$ or $K(x ; \delta)$, then $D$ either contains a free algebra over $k$ on two generators, or every finitely generated subalgebra of $D$ satisfies a polynomial identity. As a corollary, we show that the quotient division ring of any iterated Ore extension of an affine PI domain over $k$ is either again PI, or else it contains a free algebra over its center on two variables.

\section{Introduction}

Many authors have noted that it is often the case that noncommutative division algebras have free subobjects. For example, the existence of nonabelian free groups inside the multiplicative group $D^{\times}$of a division algebra $D$ has been studied in several papers (see [Reichstein and Vonessen 1995; Chiba 1996] and the references therein). It is now known that if $D$ is noncommutative and has uncountable center, then $D^{\times}$contains a free subgroup on two generators [Chiba 1996].

The question of when a division $k$-algebra $D$ contains a free $k$-subalgebra on two generators has also attracted much attention. The first result in this direction was obtained by Makar-Limanov [1983], who showed that if

$$
A_{1}(k)=k\{x, y\} /(x y-y x-1)
$$

is the Weyl algebra over a field $k$ of characteristic 0 , then its quotient division algebra $D_{1}(k)$ does indeed contain such a free subalgebra. This result is perhaps surprising to those only familiar with localization in the commutative setting, and is in fact a good demonstration of how noncommutative localization is less well-behaved. In particular, the Weyl algebra $A$ is an algebra of quadratic growth; that is, if we let $V$ denote the $k$-vector subspace of $A$ spanned by 1 and the images of $x$ and $y$

Bell was supported by NSERC grant 31-611456. Rogalski was supported by NSF grant DMS0900981.

MSC2010: primary 16K40; secondary 16S10, 16S36, 16S85.

Keywords: free algebra, division algebra, Ore extension, skew polynomial ring. 
in $A$, then the dimension of $V^{n}$ is a quadratic function of $n$. On the other hand, a free algebra on two generators has exponential growth. This is a good example of the principle that there is no nice relationship, in general, between the growth of a finitely generated algebra and the growth of other subalgebras of its quotient division algebra.

We note that by Lemma 1 of [Makar-Limanov and Malcolmson 1991], if a division $k$-algebra $D$ contains a free $k$-subalgebra on two generators, then it contains a free $F$-subalgebra on two generators for any central subfield $F$. Thus the choice of base field is not an important consideration when considering the existence of free subalgebras, and need not even be mentioned. Now there are certain division algebras which cannot contain copies of free algebras on more than one generator for trivial reasons, for example, division algebras which are algebraic over their centers. Note also that a free algebra on two generators does not satisfy a polynomial identity. We say that a $k$-algebra $R$ is locally $P I$ if every finitely generated $k$-subalgebra of $R$ is a polynomial identity ring (this is also easily seen to be independent of the choice of central base field $k$ ). An obvious necessary condition for a division algebra $D$ to contain a noncommutative free algebra is that $D$ not be locally PI. On the other hand, there are no known examples of division algebras which do not contain a free algebra on two generators, except locally PI ones.

In light of the discussion above, we say that a division algebra $D$ satisfies the free subalgebra conjecture if $D$ contains a free subalgebra on two generators if and only if $D$ is not locally PI. Makar-Limanov [1984a] annunciated the FOFS (full of free subobjects) conjecture, one part of which was the statement that every division algebra $D$ which is finitely generated (as a division algebra) and infinitedimensional over its center contains a free subalgebra on two generators. It is easy to see, using a bit of PI theory, that this statement is equivalent to what we have called the free subalgebra conjecture here. Toby Stafford independently formulated a similar conjecture, as we have learned from Lance Small. As Makar-Limanov also notes, the conjecture is a bit provocative as stated, because it implies the resolution of the Kurosh problem for division rings. However, we will study the conjecture here only for special types of division rings in any case.

Since Makar-Limanov's original breakthrough, many authors have used his ideas to demonstrate the existence of free subalgebras on two generators in the quotient division algebras of many special classes of rings, especially certain Ore extensions, group algebras, and enveloping algebras of Lie algebras [Figueiredo et al. 1996; Lichtman 1999; Lorenz 1986; Makar-Limanov 1983; 1984b; 1984c; Makar-Limanov and Malcolmson 1991; Shirvani and Gonçalves 1998; 1999]. Our main aim here is to further develop the Ore extension case. Suppose that $D$ is a division ring with automorphism $\sigma: D \rightarrow D$ and $\sigma$-derivation $\delta$, and let $D(x ; \sigma, \delta)$ be the quotient division ring of the Ore extension $D[x ; \sigma, \delta]$. Lorenz [1986] showed 
that $k(t)(x ; \sigma)$ contains a free subalgebra on two generators when $\sigma$ has infinite order. Shirvani and J. Z. Gonçalves [1999] showed that if $R$ is a $k$-algebra which is a UFD with field of fractions $K$ and the property that $R^{\times}=k^{\times}$, and $\sigma: R \rightarrow R$ is a $k$-automorphism such that the $\sigma$-fixed subring of $R$ is $k$, then $K(x ; \sigma)$ contains a free subalgebra on two generators (in fact, even a free group algebra of rank $|k|$ ).

In this paper, we first give in Section 2 some new criteria for the existence of a free $k$-subalgebra on two generators in a division ring $D(x ; \sigma, \delta)$, following the main idea of Makar-Limanov's original method. We then use these criteria to completely settle the free subalgebra conjecture for the case of Ore extensions of fields, assuming an uncountable base field. Our main results are the following.

Theorem 1.1. Let $K / k$ be a field extension and $\sigma: K \rightarrow K$ a $k$-automorphism.

(1) If $k$ is uncountable, then the following are equivalent:

(i) $K(x ; \sigma)$ contains a free $k$-subalgebra on two generators.

(ii) $K(x ; \sigma)$ is not locally PI.

(iii) $K$ has an element lying on an infinite $\sigma$-orbit.

(2) If $k$ is countable, the same conclusion as in (1) holds if either $K / k$ is infinitely generated as a field extension, or if $\sigma$ is induced by a regular $k$-automorphism of a quasiprojective $k$-variety with function field $K$.

We expect that the free subalgebra conjecture for $K(x ; \sigma)$ is always true, with no restrictions on $k$; in any case, the theorem above certainly covers the cases one is most likely to encounter.

We also study the derivation case, which is in fact easier and requires no assumption on the base field.

Theorem 1.2. Let $K$ be a field extension of a field $k$. If $\delta: K \rightarrow K$ is a $k$-derivation, then $K(x ; \delta)$ contains a free $k$-subalgebra on two generators if and only if it is not locally PI.

See Theorem 4.1 for a characterization of when $K(x ; \delta)$ is locally PI.

In fact, a general Ore extension $K[x ; \sigma, \delta]$ of a field $K$ is isomorphic to one with either $\sigma=1$ or with $\delta=0$. So as a rather quick consequence of the theorems above, we obtain the following result.

Theorem 1.3. The quotient division algebra of any iterated Ore extension of a PI domain which is affine over an uncountable field satisfies the free subalgebra conjecture.

We note that our proofs are largely independent of past work in this subject, except that we assume Makar-Limanov's original result. Some authors have considered the more general question of the existence of $k$-free group algebras in a division ring $D$, and have also studied the cardinality of the rank of the largest such free group 
algebra. For simplicity, we stick to the context of the free subalgebra conjecture here. We mention that Shirvani and Gonçalves [1996] have shown that if the center $k$ of $D$ is uncountable, then the existence of a free group $k$-algebra of rank 2 in $D$ is implied by the existence of a free $k$-algebra on two generators.

In this paper, we have tried to make as few assumptions as possible on the ground field $k$. In a follow-up paper [Bell and Rogalski 2011], we give stronger criteria for existence of free subalgebras, and thus verify the free subalgebra conjecture for some additional classes of algebras, in case $k$ is uncountable.

\section{Criteria for existence of free subalgebras of a division algebra}

In this section we use ideas of Makar-Limanov to give a simple criterion that guarantees that a division algebra $D$ contains a copy of a free algebra on two generators. We work over an arbitrary field $k$. As we noted in the introduction, the question of whether $D$ contains a free $k$-subalgebra on two generators is independent of the choice of central subfield $k$.

Notation 2.1. We use the following notation:

(1) We let $D$ denote a division algebra over a field $k$.

(2) We let $\sigma: D \rightarrow D$ denote a $k$-algebra automorphism of $D$.

(3) We let $\delta: D \rightarrow D$ denote any $\sigma$-derivation of $D$ over $k$, that is, a $k$-linear map satisfying $\delta(a b)=\sigma(a) \delta(b)+\delta(a) b$ for all $a, b \in D$.

(4) We let $\psi=(\sigma-1)+\delta: D \rightarrow D$ (this is also a $\sigma$-derivation) and set

$$
E=\{u \in D: \psi(u)=0\},
$$

which is a division subring of $D$.

(5) We let $D[x ; \sigma, \delta]$ be the Ore extension generated by $D$ and the indeterminate $x$ with relations $x a=\sigma(a) x+\delta(a)$ for $a \in D$, and let $D(x ; \sigma, \delta)$ denote its quotient division algebra. As usual, if $\sigma=1$ we omit $\sigma$ from the notation, and if $\delta=0$ we omit $\delta$ from the notation.

We now prove a sufficient condition for the ring $D(x ; \sigma, \delta)$ to contain a free subalgebra. Compared to the original method of Makar-Limanov's, we choose a slightly different pair of elements, and we avoid the use of power series. We note that the characteristic of the base field has no effect in the following criterion.

Theorem 2.2. Assume Notation 2.1 and let $b \in D$. If

(1) $b \notin \sigma(E)$, and

(2) for all $u \in D, \psi(u) \in \sigma(E)+\sigma(E) b$ implies $u \in E$,

then the $k$-algebra generated by $b(1-x)^{-1}$ and $(1-x)^{-1}$ is a free subalgebra of $D(x ; \sigma, \delta)$. 
Proof. Let

$$
\mathscr{S}=\left\{\left(i_{1}, \ldots, i_{r}\right): r \geq 1, i_{1}, \ldots, i_{r} \in\{0,1\}\right\} \cup\{\varnothing\},
$$

and for nonempty $I=\left(i_{1}, \ldots, i_{r}\right) \in \mathscr{Y}$, define length $(I):=r$ and

$$
W_{I}:=b^{i_{1}}(1-x)^{-1} b^{i_{2}}(1-x)^{-1} \cdots b^{i_{r}}(1-x)^{-1} .
$$

If $I=\varnothing$, we define length $(\varnothing):=0$ and $W_{\varnothing}:=1$. Note that the $W_{I}$ are exactly the words in the generators $b(1-x)^{-1},(1-x)^{-1}$, and so our task is to show that $\left\{W_{I} \mid I \in \mathscr{T}\right\}$ is linearly independent over $k$. It is also useful to define

$$
V_{I}:=(1-x)^{-1} b^{i_{1}}(1-x)^{-1} \cdots b^{i_{r}}(1-x)^{-1},
$$

for nonempty $I \in \mathscr{Y}$, and to set $V_{\varnothing}:=(1-x)^{-1}$.

For nonempty $I=\left(i_{1}, i_{2}, \ldots, i_{r}\right)$, we define its truncation as $I^{\prime}=\left(i_{2}, \ldots, i_{r}\right)$, with the convention that if $I$ has length 1 , then $I^{\prime}=\varnothing$. Note then that trivially from the definitions we have

$$
(1-x) V_{I}=W_{I}=b^{i_{1}} V_{I^{\prime}}, \quad \text { for } I \neq \varnothing .
$$

We claim that to prove that the $W_{I}$ are $k$-independent. It is enough to prove that $\left\{V_{I} \mid I \in S\right\}$ is left $D$-independent. To see this, suppose the $V_{I}$ are $D$-independent and that we have a nontrivial relation $\sum_{I \in S} c_{I} W_{I}=0$, with $c_{I} \in k$ not all 0 . We can assume that $c_{\varnothing}=0$ by multiplying our relation through on the right by $(1-x)^{-1}$. Then

$$
0=\sum_{I \in S} c_{I} W_{I}=\sum_{I \neq \varnothing} c_{I} b^{i_{1}} V_{I^{\prime}}=0
$$

This forces, for each nonempty $I \in S$, the equation $c_{I} b^{i_{1}}+c_{H} b^{1-i_{1}}=0$, where $H$ is the other element of $S$ which has truncation $I^{\prime}$. But then $c_{I}=c_{H}=0$, since $\{1, b\}$ is certainly $k$-independent, given that $b \notin \sigma(E)$. This contradicts the nontriviality of our chosen relation and the claim is proved.

The strategy is to prove by contradiction that $\left\{V_{I} \mid I \in S\right\}$ is left $D$-independent. In fact, it is more convenient to prove the seemingly stronger statement that this set is $D$-independent in the left factor $D$-space $D(x ; \sigma, \delta) / D[x ; \sigma, \delta]$. In other words, we work modulo polynomials. Equivalently, we suppose that we have a relation $\sum_{I \in S} \alpha_{I} V_{I}=p(x) \in D[x ; \sigma, \delta]$, with $\alpha_{I} \in D$ not all 0 . Among all such relations, we pick one with a minimal value of $d=\min \left(\right.$ length $(I) \mid \alpha_{I} \neq 0$ ). Moreover, among these, we select one with the smallest number of nonzero $\alpha_{I}$ with length $(I)=d$. Note that certainly $d \geq 1$. By multiplying our relation by a nonzero element of $D$, we may also assume that $\alpha_{J}=1$, for some $J$ of length $d$.

Now for nonempty $I,(2.4)$ can be rewritten as $x V_{I}=V_{I}-b^{i_{1}} V_{I^{\prime}}$, and for $I=\varnothing$, we have $x V_{I}=V_{I}-1$. Multiplying our relation on the left by $x$ and applying these 
formulas, we obtain

$$
\begin{aligned}
x \sum_{I \in S} \alpha_{I} V_{I} & =\sum_{I \in S}\left[\sigma\left(\alpha_{I}\right) x+\delta\left(\alpha_{I}\right)\right] V_{I} \\
& =\sum_{I \in S}\left[\sigma\left(\alpha_{I}\right)+\delta\left(\alpha_{I}\right)\right] V_{I}-\sum_{I \neq \varnothing} \sigma\left(\alpha_{I}\right) b^{i_{1}} V_{I^{\prime}}-\sigma\left(\alpha_{\varnothing}\right) \\
& =x p(x) \in D[x ; \sigma, \delta] .
\end{aligned}
$$

Subtracting the original relation $\sum \alpha_{I} V_{I}=p(x)$, we get

$$
\sum_{I \in S}\left[\sigma\left(\alpha_{I}\right)-\alpha_{I}+\delta\left(\alpha_{I}\right)\right] V_{I}-\sum_{I \neq \varnothing} \sigma\left(\alpha_{I}\right) b^{i_{1}} V_{I^{\prime}}=(x-1) p(x)+\sigma\left(\alpha_{\varnothing}\right) \in D[x ; \sigma, \delta] .
$$

But notice that since $\alpha_{J}=1$, the coefficient of $V_{J}$ in this relation is now 0 , while no new nonzero coefficients associated to $V_{I}$ with $I$ of length $d$ have appeared. Thus by our assumption that we originally picked a minimal relation, all coefficients of the $V_{I}$ on the left-hand side of (2.5) are 0 . In particular,

$$
\psi\left(\alpha_{I}\right)=\sigma\left(\alpha_{I}\right)-\alpha_{I}+\delta\left(\alpha_{I}\right)=0,
$$

so $\alpha_{I} \in E$, for all $I$ of length $d$. Also, if $H$ is the other element of $S$ with truncation $J^{\prime}$, then the coefficient of $V_{J^{\prime}}$ in (2.5) is

$$
\sigma\left(\alpha_{J^{\prime}}\right)-\alpha_{J^{\prime}}+\delta\left(\alpha_{J^{\prime}}\right)-\sigma\left(\alpha_{J}\right) b^{j_{1}}-\sigma\left(\alpha_{H}\right) b^{1-j_{1}}=0 .
$$

Since $H$ and $J$ have length $d$, for $u=\alpha_{J^{\prime}}$ we obtain

$$
\psi(u)=\sigma(u)-u+\delta(u) \in \sigma(E) b+\sigma(E) .
$$

Note that also $\psi(u) \neq 0$, as the assumption $b \notin \sigma(E)$ implies that $\sigma(E) b+\sigma(E)$ is direct, and $\sigma\left(\alpha_{J}\right)=1$. The existence of such a $u$ violates the hypothesis, so we have achieved a contradiction. Thus $b(1-x)^{-1}$ and $(1-x)^{-1}$ generate a free subalgebra of $D(x ; \sigma, \delta)$, as claimed.

The interaction between $\delta$ and $\sigma$ in the criterion of the preceding theorem seems to make it hard to analyze in general. In practice, we will only use the theorem later in the special cases where $\delta=0$ or $\sigma=1$. In the rest of this section, we examine the criterion for the special case of $D(x ; \sigma)$ more closely. As mentioned in the introduction, Makar-Limanov [1983] proved that the Ore quotient ring of the first Weyl algebra, $D_{1}(k)$, contains a free $k$-subalgebra on two generators when $k$ has characteristic 0 (see also [Krause and Lenagan 2000, Theorem 8.17]). It is standard that $D_{1}(k) \cong k(u)(x ; \sigma)$, where $\sigma(u)=u+1$, but we note that Theorem 2.2, as stated, does not recover Makar-Limanov's result. More specifically, taking $D=k(u)$, $\sigma(u)=u+1$, and $\delta=0$ in Notation 2.1, it is easy to see that $E=k$, but we have $\sigma(u)-u \in k$ with $u \notin k$; thus the criterion in Theorem 2.2 cannot be satisfied 
regardless of $b$. In fact, our criterion seems to be most useful when we combine it with Makar-Limanov's known result to give the following stronger criterion.

Theorem 2.3. Assume the notation from Notation 2.1, with $\delta=0$. Suppose that either $k$ has characteristic 0 , or else $k$ has characteristic $p>0$ and we have the additional condition that

$$
\left\{a \in D \mid \sigma^{p}(a)=a\right\}=E .
$$

If there is $b \in D \backslash E$ such that the equation

$$
\sigma(u)-u \in b+E
$$

has no solutions for $u \in D$, then the $k$-algebra generated by $b(1-x)^{-1}$ and $(1-x)^{-1}$ is a free subalgebra of $D(x ; \sigma)$.

Proof. Choose $b$ as in the hypothesis. If $\sigma(u)-u \in E+E b$ has no solutions except for $u \in E$, then we are done by Theorem 2.2. So we may assume there is a solution of the form $\sigma(u)-u=\alpha+\beta b$ with $\alpha, \beta \in E$ not both zero. As long as $\beta \neq 0$, we may replace $u$ by $\beta^{-1} u$ and thus assume that $\beta=1$, and so (2.6) has a solution, contradicting the hypothesis. Thus $\beta=0$. Then $\alpha \neq 0$ and $y=u \alpha^{-1}$ satisfies $\sigma(y)=y+1$. Then the elements $z=y x^{-1}$ and $x$ satisfy the relation $x z-z x=1$. If char $k=0$, then we see that the $k$-subalgebra $R$ of $D(x ; \sigma)$ generated by $x$ and $z$ is isomorphic to a factor of the Weyl algebra $A_{1}(k)$. Since the Weyl algebra is simple, $R \cong A_{1}(k)$, and so $D(x ; \sigma)$ must contain a copy of $D_{1}(k)$, and hence a free $k$-algebra on two generators [Makar-Limanov 1983]. If instead char $k=p>0$, then we have $\sigma^{p}(y)=y$. It follows by the hypothesis that $y \in E$, but this contradicts $\sigma(y)=y+1$.

We end this section with a valuation-theoretic criterion that will be especially useful later when $D$ is a field. Recall that a discrete valuation of a division ring $D$ is a function $v: D^{\times} \rightarrow \mathbb{Z}$ such that $v(x y)=v(x)+v(y)$ and $v(x+y) \geq \min (v(x), v(y))$ for all $x, y \in D^{\times}$. It is easy to see that $v(x+y)=\min (v(x), v(y))$ if $v(x) \neq v(y)$. The valuation $v$ is trivial if $v(x)=0$ for all $x \in D^{\times}$.

Lemma 2.4. Assume the notation from Notation 2.1, with $\delta=0$. Suppose that $D$ has a nontrivial discrete valuation $v: D^{\times} \rightarrow \mathbb{Z}$, such that $(i) v(a)=0$ for all $a \in E$; and (ii) for all $a \in D^{\times}, v\left(\sigma^{n}(a)\right)=0$ for all $n \gg 0$ and all $n \ll 0$. If char $k=0$, or if char $k=p>0$ and $\left\{y \in D \mid \sigma^{p}(y)=y\right\}=E$, then $D(x ; \sigma)$ contains a free subalgebra on two generators.

Proof. For any given $u \in D$, by hypothesis $X_{u}=\left\{n \in \mathbb{Z} \mid v\left(\sigma^{n}(u)\right)<0\right\}$ is a finite set, and if $X_{u} \neq \varnothing$, we call $\ell(u)=\max X_{u}-\min X_{u}$ the length of $u$. If $X_{u} \neq \varnothing$, then it is easy to see that $\ell(u-\sigma(u))=\ell(u)+1$.

By nontriviality, we can pick $b \in D$ such that $X_{b} \neq \varnothing$. Among all such $b$, choose one of minimal length, say $\ell(b)=d$. We claim that there are no solutions to the 
equation $u-\sigma(u)=b+e$ with $u \in D$ and $e \in E$. Suppose $(u, e)$ does give such a solution. It is easy to see that $\ell(b+e)=\ell(b)=d$, since $v\left(\sigma^{n}(e)\right)=0$ for all $n$ by hypothesis (i). Now $X_{u}=\varnothing$ is clearly impossible, so $u$ has a length. By minimality, $\ell(u) \geq d$, and so $\ell(u-\sigma(u)) \geq d+1$, a contradiction. The result now easily follows from Theorem 2.3.

\section{The automorphism case}

In this section, the goal is to use the criteria developed in the previous section to study when $K(x ; \sigma)$ contains a free subalgebra, where $K$ is a field, and thus to prove Theorem 1.1. In terms of Notation 2.1, we now write $D=K$ for a field $K$ containing the base field $k$ with $k$-automorphism $\sigma: K \rightarrow K$, and assume that $\delta=0$. Then $K(x ; \sigma)$ is also an algebra over the fixed subfield $E=\{a \in K \mid \sigma(a)=a\}$, and as already mentioned, we may change the base field to any central subfield without affecting the question of the existence of free subalgebras. Thus, it does no harm to replace $k$ by $E$, and we assume that the base field $k$ is the $\sigma$-fixed field for the rest of this section. We will frequently use in this section the exponent notation $b^{\sigma}:=\sigma(b)$ for the action of an automorphism on an element.

The difficult direction of Theorem 1.1 is to prove that $K(x ; \sigma)$ contains a free subalgebra on two generators if $K$ contains an element $a$ lying on an infinite $\sigma$ orbit. In this case, letting $K^{\prime}=k\left(\ldots, a^{\sigma^{-2}}, a^{\sigma^{-1}}, a, a^{\sigma}, \ldots\right)$ be the subfield of $K$ generated over $k$ by the $\sigma$-orbit of $a$, it suffices to prove that $K^{\prime}(x ; \sigma)$ contains a noncommutative free subalgebra. Thus, in this section, we will often assume the following hypothesis.

Hypothesis 3.1. Let $K$ be a field with automorphism $\sigma: K \rightarrow K$, and let $k$ be the fixed field of $\sigma$. Assume that there is an element $a \in K$ on an infinite $\sigma$-orbit such that $K=k\left(a^{\sigma^{n}} \mid n \in \mathbb{Z}\right)$.

The proof that $K(x ; \sigma)$ satisfying Hypothesis 3.1 contains a free subalgebra naturally breaks up into two cases, depending on whether or not $K / k$ is finitely generated as a field extension. The infinitely generated case is rather easily dispatched. We thank the referee very much for suggesting the elegant proof of the following proposition, which gives a simpler and more direct method for handling the infinitely generated case than our original.

Proposition 3.2. Assume Hypothesis 3.1, and suppose that $K$ is infinitely generated as a field extension of $k$. Then $K(x ; \sigma)$ contains a free $k$-subalgebra on two generators.

Proof. Write $a_{j}=a^{\sigma^{j}}$ for all $j \in \mathbb{Z}$. Suppose first that $k\left(a_{i} \mid i \geq 0\right)$ is still an infinitely generated field extension of $k$. In this case, we will show that in fact 
the countable system of elements $\left\{a x^{j} \mid j \geq 1\right\}$ generates a free $k$-subalgebra of $K(x ; \sigma)$.

Let $y_{j}=a x^{j}$ for all $j \geq 1$. Then an arbitrary monomial in the $y_{j}$ looks like

$$
y_{j_{1}} y_{j_{2}} \ldots y_{j_{n}}=a_{0} a_{j_{1}} a_{j_{1}+j_{2}} \ldots a_{j_{1}+j_{2}+\cdots+j_{n-1}} x^{j_{1}+j_{2}+\cdots+j_{n}}
$$

for some $j_{1}, \ldots, j_{n} \geq 1$. We claim that the set

$$
S=\left\{a_{0} a_{i_{1}} a_{i_{2}} \ldots a_{i_{m}} \mid m \geq 1,0<i_{1}<i_{2}<\cdots<i_{m}\right\} \cup\left\{a_{0}\right\}
$$

is linearly independent over $k$. Suppose not, and pick a linear dependency relation over $k$ in which the maximum $n$ such that $a_{n}$ appears in this relation is as small as possible. Clearly $n \geq 1$. Since every element of $S$ is a product of distinct $a_{i}$, the dependency relation has the form $p a_{n}+q=0$, where $p, q$ are linear combinations of elements of $S$ involving only $a_{i}$ with $i<n$. If $p=0$, we contradict the choice of $n$. Thus $a_{n}=q / p \in k\left(a_{0}, a_{1}, \ldots, a_{n-1}\right)$. Applying $\sigma$, this easily implies by induction that $k\left(a_{0}, a_{1}, \ldots, a_{n-1}\right)=k\left(a_{i} \mid i \geq 0\right)$, contradicting the assumption that the latter field is infinitely generated as an extension of $k$. This establishes the claim that $S$ is linearly independent over $k$. Together with the $K$-independence of the powers of $x$, this implies that the distinct monomials $y_{j_{1}} y_{j_{2}} \ldots y_{j_{n}}$ are linearly independent over $k$. In other words, the $y_{i}$ generate a free subalgebra of $K(x ; \sigma)$ as required.

Suppose instead that $k\left(a_{i} \mid i \leq 0\right)$ is an infinitely generated field extension of $k$. A symmetric argument to the above shows that $\left\{a x^{j} \mid j \leq-1\right\}$ generates a free subalgebra of $K(x ; \sigma)$. Finally, if both $k\left(a_{i} \mid i \leq 0\right)$ and $k\left(a_{i} \mid i \geq 0\right)$ are finitely generated field extensions of $k$, then $K=k\left(a_{i} \mid i \in \mathbb{Z}\right)$ is also a finitely generated extension of $k$, contradicting the hypothesis.

Now we begin to tackle the case where $K / k$ is a finitely generated field extension. The idea in this case is to construct an appropriate valuation satisfying the hypothesis of Lemma 2.4, using algebraic geometry, which we do in the next proposition. All geometric notions we use will be standard ones as defined in [Hartshorne 1977]. Though sometimes we cite passages in that reference that belong to sections with a blanket hypothesis that the base field $k$ is algebraically closed, one can check in each case that this restriction is unnecessary.

Before proving the proposition, we review some basic geometric facts that we will need in particular, and prove a lemma. For convenience, we work only with quasiprojective varieties over the field $k$, as defined in [Hartshorne 1977, Section I.2], and for brevity we simply call these $k$-varieties. While for the most part we do not need schemes, it will be convenient occasionally to think of a variety as a scheme, as in [Hartshorne 1977, Proposition II.2.6], so that we may use the notion of the generic point of an irreducible subvariety. 
Let $X$ be a $k$-variety. Recall that there is a bijective correspondence between birational maps $\phi: X \rightarrow X$ and automorphisms of the field $k(X)$ of rational functions on $X$ [Hartshorne 1977, Theorem I.4.4]. More explicitly, given a birational map $\phi: X \rightarrow X$, for $f \in k(X)$ we write $\phi(f)=f^{\phi}=f \circ \phi \in k(X)$; then $f \mapsto f^{\phi}$ is the corresponding automorphism of $k(X)$, and we call this automorphism $\phi: k(X) \rightarrow k(X)$ as well. Conversely, if we begin with an automorphism $\tau: K \rightarrow K$ of a finitely generated field extension $K / k$, we may be able to choose a $k$-variety $X$ with function field $k(X)=K$ such that the corresponding birational map $\tau: X \rightarrow X$ is not just birational, but in fact a regular $k$-automorphism of $X$. In this case we say that $\tau: K \rightarrow K$ is induced by a regular $k$-automorphism of $X$. In general, however, it could be that no such choice of $X$ exists; see, for example, [Rogalski 2009, Section 3] for more discussion of this issue.

Suppose that $X$ is a normal $k$-variety. Then for any prime divisor (that is, codimension- 1 irreducible subvariety) $C$ of $X$, there is a corresponding discrete valuation $v_{C}$ of $K=k(X)$ such that $v_{C}(f)$ is the order of vanishing of $f$ along $C$ [Hartshorne 1977, Section II.6]. Recall that Div $X$ is the free abelian group with basis the distinct prime divisors $C$ on $X$. Given a rational function $f \in k(X)$, we define its corresponding principal divisor in Div $X$ as

$$
(f)=\sum_{C} v_{C}(f) C .
$$

Now suppose that $\sigma: X \rightarrow X$ is a birational map of a normal projective $k$-variety. Then by Zariski's main theorem, the closed set where $\sigma$ is undefined is at least codimension 2 in $X$. In other words, given any prime divisor $C$ on $X, \sigma$ is defined at the generic point $\eta$ of $C$ [Hartshorne 1977, Lemma V.5.1], or equivalently, $\sigma$ is defined on an open subset of the points of $C$. We write $\sigma(C)$ to mean the closure of $\sigma(\eta)$. Since $\sigma$ is merely birational, $\sigma(C)$ may be a closed subset of codimension greater than 1 in $X$, in which case we say that $\sigma$ contracts $C$. On the other hand, if $\sigma(C)$ is again of codimension 1 , then the birational map $\sigma^{-1}$ must be defined at the generic point $\sigma(\eta)$ of $\sigma(C)$, and we conclude that $\sigma$ is a local isomorphism from an open neighborhood of $\eta$ to an open neighborhood of $\sigma(\eta)$.

Lemma 3.3. Let $\sigma: X \rightarrow X$ be a birational map of a normal projective $k$-variety. If $C$ is a prime divisor not contracted by $\sigma$, then $v_{\sigma(C)}=v_{C} \circ \sigma$. Moreover, if $f \in K=k(X)$ has the property that $\sigma^{-1}$ contracts no prime divisor appearing with nonzero coefficient in $D=(f)$, and $\sigma$ contracts no prime divisor appearing with nonzero coefficient in $E=\left(f^{\sigma}\right)$, then $E=\sigma^{-1}(D)$.

Proof. Let $C$ be a prime divisor not contracted by $\sigma$, so that $\sigma$ is a local isomorphism from a neighborhood of the generic point $\eta$ of $C$ to a neighborhood of the generic point $\sigma(\eta)$ of $\sigma(C)$. The formula $v_{\sigma(C)}=v_{C} \circ \sigma$ follows, since by definition, 
$v_{\sigma(C)}(f)$ depends only on the image of $f$ in the local ring $\mathscr{O}_{X, \sigma(\eta)}$ of functions defined in a neighborhood of $\sigma(\eta)$.

For the second statement, suppose that $C$ is a prime divisor such that $v_{C}(f) \neq 0$. Then by hypothesis, $\sigma^{-1}$ does not contract $C$, and so $B=\sigma^{-1}(C)$ is another prime divisor which is not contracted by $\sigma$, and using the previous paragraph, we have that $v_{B}\left(f^{\sigma}\right)=v_{\sigma(B)}(f)=v_{C}(f) \neq 0$. Conversely, if $B$ is a prime divisor with $v_{B}\left(f^{\sigma}\right) \neq 0$, then by hypothesis, $C=\sigma(B)$ is another prime divisor and again $v_{C}(f)=v_{\sigma(B)}(f)=v_{B}\left(f^{\sigma}\right) \neq 0$. It follows that if $B_{1}, \ldots, B_{m}$ are the distinct prime divisors occurring with nonzero coefficient in $\left(f^{\sigma}\right)$, then $C_{1}=\sigma\left(B_{1}\right), \ldots, C_{m}=$ $\sigma\left(B_{m}\right)$ are the distinct prime divisors occurring with nonzero coefficient in $(f)$. Thus

$$
\begin{aligned}
E & =\left(f^{\sigma}\right)=\sum_{i=1}^{m} v_{B_{i}}\left(f^{\sigma}\right) B_{i}=\sum_{i=1}^{m} v_{\sigma\left(B_{i}\right)}(f) B_{i} \\
& =\sum_{i=1}^{m} v_{C_{i}}(f) \sigma^{-1}\left(C_{i}\right)=\sigma^{-1}\left(\sum_{i=1}^{m} v_{C_{i}}(f) C_{i}\right)=\sigma^{-1}(D),
\end{aligned}
$$

as required.

We remark that a similar (but easier) argument shows that if instead $\sigma: X \rightarrow X$ is a regular $k$-automorphism of any normal $k$-variety, then the formulas $v_{\sigma(C)}=v_{C} \circ \sigma$ and $\left(f^{\sigma}\right)=\sigma^{-1}[(f)]$ as in the previous result always hold for any prime divisor $C$ and any $f \in k(X)$.

The assumption in the next result that $K / k$ is totally transcendental (that is, that every element $b \in K \backslash k$ is transcendental over $k$ ) will be easily removed in the proof of Theorem 1.1.

Proposition 3.4. Assume Hypothesis 3.1, and that $K / k$ is a totally transcendental finitely generated field extension of $k$. Suppose that either $k$ is uncountable, or that there is a quasiprojective $k$-variety $X$ with function field $K$ such that $\sigma: K \rightarrow K$ is induced by a regular $k$-automorphism of $X$. Then $K(x ; \sigma)$ contains a free subalgebra on two generators.

Proof. Note that if an element $b$ in $K$ has finite order under $\sigma$, it is algebraic over $k$, and thus in $k$ by the assumption that $K / k$ is totally transcendental. Thus all elements in $K / k$ have infinite order under $\sigma$. In particular, $K / k$ must have transcendence degree at least 1 .

Assume first that there is a quasiprojective $k$-variety $X$ with function field $K$ such that $\sigma: K \rightarrow K$ is induced by a regular $k$-automorphism of $X$, which we give the same name $\sigma: X \rightarrow X$. An automorphism of a variety induces an automorphism of its normalization, by the universal property of the normalization [Hartshorne 1977, Exercise II.3.8]. Thus by replacing $X$ with its normalization (which is again quasiprojective by a standard result), we can assume that $X$ is normal. Suppose 
that $X$ has an prime divisor $C$ lying on an infinite $\sigma$-orbit of divisors. Then we take the valuation $v_{C}$ of $K$, which satisfies the hypotheses of Lemma 2.4 since $v_{\sigma^{i}(C)}=v_{C} \circ \sigma^{i}$ by the remark following Lemma 3.3, and since a rational function has a pole or zero along at most finitely many of the divisors $\sigma^{i}(C)$. We conclude by that lemma that $K(x ; \sigma)$ contains a free $k$-algebra on two generators. (Note that the extra hypothesis of Lemma 2.4 in characteristic $p$ holds since $K / k$ is totally transcendental.)

Otherwise, all prime divisors $C$ of $X$ lie on finite $\sigma$-orbits. We now apply a similar argument as in [Bell et al. 2010, Theorem 5.7] to show that this implies the existence of a $\sigma^{n}$-eigenvector in $K \backslash k$ for some $n$. Note that $X$ certainly has infinitely many distinct prime divisors, since $\operatorname{dim} X \geq 1$. Thus we can pick a sequence of rational functions $f_{1}, f_{2}, \cdots \in K \backslash k$ such that for each $i, f_{i}$ has a zero or pole along some prime divisor not appearing in $\left(f_{j}\right)$ for all $1 \leq j<i$. Note that for any $i$, if $n$ is a multiple of the order under $\sigma$ of all of the divisors appearing in $\left(f_{i}\right)$, then $\left(f_{i}^{\sigma^{n}}\right)=\sigma^{-n}\left[\left(f_{i}\right)\right]=\left(f_{i}\right)$ (using the remark following Lemma 3.3), and thus $u_{i}=f_{i}^{\sigma^{n}} / f_{i}$ has principal divisor $\left(u_{i}\right)=0$. In other words, $u_{i}$ is in $G=\Gamma\left(X, O_{X}\right)^{*}$, the units group of the ring of global regular functions on $X$. Now $H=G /\left(\bar{k}^{*} \cap G\right)$ is a finitely generated abelian group [Bell et al. 2010, Lemma 5.6(2)] which is easily seen to be torsion-free, where $\bar{k}$ is the algebraic closure of $k$. (We note that in order to apply this result of Bell et al., we need $X$ to be quasiprojective.) In fact, since $K / k$ is totally transcendental, we have that $H=G /\left(k^{*} \cap G\right)$; say this group has rank $d$. Then we can choose $n>0$ such that $\left(f_{i}^{\sigma^{n}}\right)=\left(f_{i}\right)$ for all $1 \leq i \leq d+1$, and so $u_{i}=f_{i}^{\sigma^{n}} / f_{i}$ is in $G$ for all $1 \leq i \leq d+1$. This forces $\lambda=u_{1}^{a_{1}} u_{2}^{a_{2}} \ldots u_{d+1}^{a_{d+1}} \in k$ for some integers $a_{i}$, not all 0 . Then $g=f_{1}^{a_{1}} f_{2}^{a_{2}} \ldots f_{d+1}^{a_{d+1}}$ satisfies $\sigma^{n}(g)=\lambda g$. Moreover, $g \notin k$, because otherwise $\left(f_{d+1}\right)$ would involve only prime divisors occurring among the $\left(f_{i}\right)$ with $1 \leq i<d+1$. Now if $\lambda$ is a root of 1 , then $\sigma^{m}(g)=g$ for some $m>0$, which implies that $g$ is algebraic over $k$, contradicting that $K / k$ is totally transcendental. So $\lambda$ has infinite multiplicative order. Then $L=k(g)$ is a rational function field over $k$ to which the automorphism $\sigma^{n}$ restricts as an infinite order automorphism, and it suffices to show that $L\left(x ; \sigma^{n}\right)$ contains a free subalgebra on two generators. This follows from another application of Lemma 2.4 to $L$ and its automorphism $\left.\sigma^{n}\right|_{L}$, choosing the valuation associated to the maximal ideal $(g-1)$ of $k[g]$, which lies on an infinite $\left.\sigma^{n}\right|_{L}$-orbit.

Next, we assume instead that $k$ is uncountable. In this case, we will have to work with a birational map of a variety only, but will be able to perform a similar argument to the above by choosing $f_{i}$ such that $\left(f_{i}\right)$ avoids the places where the birational map is not an isomorphism. Since $K / k$ is finitely generated, it is wellknown that we can choose a normal projective $k$-variety $X$ such that $k(X)=K$. The automorphism $\sigma: K \rightarrow K$ corresponds to a birational map $\sigma: X \rightarrow X$. Since $\sigma$ is an isomorphism on some open subset of $X, \sigma$ must contract at most finitely 
many prime divisors. Thus the set $S$ of prime divisors on $X$ which are contracted by some $\sigma^{n}$ with $n \in \mathbb{Z}$ is countable.

We now show that we can find a plentiful supply of rational functions whose $\sigma$-iterates have divisors entirely avoiding the bad set $S$. Pick any element $h \in K \backslash k$. Consider $h+\lambda$ as $\lambda \in k$ varies. The divisors along which $h+\lambda_{1}$ and $h+\lambda_{2}$ have a zero are disjoint if $\lambda_{1} \neq \lambda_{2}$. For a given $i \in \mathbb{Z}$, there are countably many $\lambda$ such that $h^{\sigma^{i}}+\lambda$ has a zero along a divisor in $S$. Since $k$ is uncountable, there are uncountably many $\lambda$ such that $h^{\sigma^{i}}+\lambda$ has zeroes only along divisors not in $S$, for all $i$. Fix such a $\lambda$ and put $g=1 /(h+\lambda)$; thus $g^{\sigma^{i}}$ has poles only along divisors not in $S$, for all $i$. By the same argument, for uncountably many $\mu$, the rational functions $g^{\sigma^{i}}+\mu$ have no zeroes in $S$, for all $i$. Let $f_{\mu}=g+\mu$. By construction, there are uncountably many $\mu \in k$ such that $f_{\mu}^{\sigma^{i}}$ has no zeroes or poles in $S$, for all $i$; and moreover, $f_{\mu_{1}}$ and $f_{\mu_{2}}$ have disjoint zeroes if $\mu_{1} \neq \mu_{2}$.

Now we may essentially repeat the argument of the first half of the proof. If there is a prime divisor $C$ not in $S$ which lies on an infinite $\sigma$-orbit, then since the equation $v_{\sigma^{i}(C)}=v_{C} \circ \sigma^{i}$ holds for all $i \in \mathbb{Z}$, by Lemma 3.3, this allows us to apply Lemma 2.4 to the valuation $v_{C}$ to conclude that $K(x ; \sigma)$ contains a free subalgebra on two generators. Otherwise, by the construction of the $f_{\mu}$, we may choose a sequence of rational functions $f_{1}, f_{2}, \ldots$ from among the uncountably many $f_{\mu}$ 's whose $\sigma$-iterates all avoid the set $S$, such that each $\left(f_{i}\right)$ has a zero along some prime divisor not appearing in $\left(f_{j}\right)$, for all $1 \leq j<i$. Lemma 3.3 implies that the equation $\left(f_{i}^{\sigma^{n}}\right)=\sigma^{-n}\left[\left(f_{i}\right)\right]=\left(f_{i}\right)$, which was needed in the third paragraph of the proof, still holds for any $n$ which is a multiple of the (necessarily finite) order under $\sigma$ of the prime divisors occurring in $\left(f_{i}\right)$. Thus, the same argument as in the third paragraph of the proof goes through to construct a free subalgebra of $K(x ; \sigma)$ in this case also.

Proof of Theorem 1.1. Let $\sigma: K \rightarrow K$ be an automorphism of $K / k$. If every element of $K$ lies on a finite $\sigma$-orbit, then setting $K_{n}=\left\{x \in K \mid \sigma^{n}(x)=x\right\}$, we will have $K=\bigcup_{n \geq 1} K_{n}$, and thus $K(x ; \sigma)=\bigcup_{n \geq 1} K_{n}(x ; \sigma)$ is a directed union of PI algebras. Thus it is locally PI, and cannot possibly contain a free subalgebra on two generators.

To complete the proof, we assume that there is an element $a \in K$ lying on an infinite $\sigma$-orbit, and need to prove that $K(x ; \sigma)$ contains a free subalgebra. We have seen that we may assume the conditions in Hypothesis 3.1, so that $k$ is the fixed field of $\sigma$ and $K=k\left(a^{\sigma^{n}} \mid n \in \mathbb{Z}\right)$. If $K / k$ is infinitely generated as a field extension, then we are done by Proposition 3.2, with no assumptions on the base field $k$ necessary. Suppose instead that $K / k$ is finitely generated, and that we have either that (i) $k$ is uncountable, or (ii) there is a $k$-automorphism $\sigma$ of a quasiprojective $k$-variety $X$ with $k(X)=K$ inducing $\sigma: K \rightarrow K$ (we may assume that $X$ is normal, just as 
in the proof of Proposition 3.2). To apply Proposition 3.4, we need to reduce to the totally transcendental case. If $L \subseteq K$ is the subfield of elements algebraic over $k$, then $L / k$ is also finitely generated, and thus $[L: k]<\infty$. The elements in $L$ have finite order under $\sigma$, and thus there is a single power $\sigma^{d}$ such that $\sigma^{d}(b)=b$ for all $b \in L$. Let $K^{\prime}:=k\left(a^{\sigma^{n d}} \mid n \in \mathbb{Z}\right)$. We now replace $(K, \sigma)$ by $\left(K^{\prime}, \sigma^{d}\right)$. By construction, $K^{\prime}$ is still generated by the $\sigma^{d}$-iterates of a single element $a$ on an infinite orbit. The field $k^{\prime}=\left\{b \in K \mid \sigma^{d}(b)=b\right\}$ certainly contains $L$; in fact, $k^{\prime}$ is algebraic over the field $k$ of $\sigma$-fixed elements, and so $k^{\prime}=L$. Thus the fixed field of $\sigma^{d}: K^{\prime} \rightarrow K^{\prime}$ is now $K^{\prime} \cap L$, and the field extension $K^{\prime} /\left(K^{\prime} \cap L\right)$ is now totally transcendental. If we have hypothesis (ii), then $X$ is an $L$-variety since rational functions which are algebraic over $k$ must be global regular functions ( $X$ is normal), and $\sigma^{d}: X \rightarrow X$ is now an $L$-automorphism. Thus in either case (i) or (ii), replacing the triple $(K / k, \sigma, a)$ with $\left(K^{\prime} /\left(K^{\prime} \cap L\right), \sigma^{d}, a\right)$ preserves the hypothesis, and now $K^{\prime} /\left(K^{\prime} \cap L\right)$ is totally transcendental. By Proposition $3.4, K^{\prime}\left(x ; \sigma^{d}\right)$ contains a free $k$-subalgebra on two generators, and thus so does the larger division algebra $K(x ; \sigma)$.

We close this section with a few remarks related to the main theorem.

Remark 3.5. Consider an arbitrary (possibly countable) base field $k$, and $k$-automorphism $\sigma: K \rightarrow K$, where $K$ contains an element $a$ lying on an infinite $\sigma$ orbit. Although our methods do not in full generality allow us to conclude that $K(x ; \sigma)$ contains a free subalgebra on two generators, we may always conclude that $K\left(t_{1}, \ldots, t_{n}\right)(x ; \sigma)$ contains a free subalgebra on two generators for some $n$, where the $t_{i}$ are commuting indeterminates and we extend $\sigma$ to $K\left(t_{1}, \ldots, t_{n}\right)$ by setting $\sigma\left(t_{i}\right)=t_{i}$ for all $i$. To see this, note that adjoining an uncountable set of indeterminates $\left\{t_{\alpha}\right\}$ to $K$, we can consider $K\left(\left\{t_{\alpha}\right\}\right)(x ; \sigma)$ as an algebra over the uncountable field $k\left(\left\{t_{\alpha}\right\}\right)$. Then Theorem 1.1 applies and shows that $K\left(\left\{t_{\alpha}\right\}\right)(x ; \sigma)$ contains a free subalgebra on two generators; but note that these generators live in $K\left(t_{1}, \ldots, t_{n}\right)(x ; \sigma)$ for some finite subset $\left\{t_{1}, \ldots, t_{n}\right\}$ of $\left\{t_{\alpha}\right\}$.

In characteristic 0 , we can do even better and conclude that $K(t)(x ; \sigma)$ contains a free subalgebra. This easily follows from an application of Lemma 2.4 to the discrete valuation $v$ of $K(t)$ corresponding to the maximal ideal $(t-a)$ of $K[t]$. In [Bell and Rogalski 2011, Theorem 2.6], we show how this idea provides an alternate proof of Theorem 1.1 when $k$ is uncountable of characteristic 0 , since when $k$ is uncountable, we prove that a division $k$-algebra $D$ contains a free subalgebra on two generators if and only if $D(t)$ does [Bell and Rogalski 2011, Proposition 2.1].

Remark 3.6. The division rings $K(x ; \sigma)$ really can be only locally PI rather than PI. For example, let $K=\mathbb{C}\left(y_{1}, y_{2}, \ldots\right)$ be a function field in infinitely many indeterminates and define an automorphism $\sigma: K \rightarrow K$ which fixes $\mathbb{C}$ and has 
$\sigma\left(y_{n}\right)=\zeta_{n} y_{n}$ for a primitive $n$-th root of unity $\zeta_{n}$. If $K_{n}=\mathbb{C}\left(y_{n}\right)$, then it is easy to check that $K_{n}(x ; \sigma)$ is a subdivision ring of $K(x ; \sigma)$ with PI degree exactly $n$.

\section{The derivation case}

Assume Notation 2.1, with $K=D$ a field extension of $k, \sigma=1$, and $\delta: K \rightarrow K$ a $k$-derivation, so $E=\{a \in K \mid \delta(a)=0\}$. In this section, we show that $K(x ; \delta)$ satisfies the free subalgebra conjecture. Since $K(x ; \delta)$ is an $E$-algebra, as usual we can and do replace the base field $k$ by the central subfield $E$. In fact, the analysis of $K(x ; \delta)$ is much easier than the automorphism case. In characteristic 0 , this reduces rather trivially to the case of the Weyl algebra (as other authors have also observed). So our main contribution here is to consider the characteristic $p$ case.

Theorem 4.1. Let $\delta: K \rightarrow K$ be a derivation of a field, where $k=\{a \in K \mid \delta(a)=0\}$.

(1) If char $k=0$, then $K(x ; \delta)$ contains a free subalgebra if and only if $\delta \neq 0$.

(2) If char $k=p>0$, then $K(x ; \delta)$ contains a free subalgebra if and only if there is an element $a \in K$ such that setting $F_{i}=k\left(a, \delta(a), \ldots, \delta^{i-1}(a)\right)$, one has $F_{i} \subsetneq F_{i+1}$ for all $i \geq 0$.

Proof. (1) If $\delta \neq 0$, say $\delta(a) \neq 0$, take $y=a$ and $z=x(\delta(a))^{-1}$. Then $y z-z y=1$, so $D$ contains a copy of the Weyl algebra $A_{1}(k)$, and hence a free algebra on two generators by Makar-Limanov's original result [1983]. On the other hand, if $\delta=0$, then $K(x ; \delta) \cong K(x)$ is commutative and cannot contain a noncommutative free subalgebra.

(2) Since char $k=p$, note that $\delta\left(b^{p}\right)=0$ for all $b \in K$, so $k$ contains all $p$-th powers. Now fix $a \in K$ and consider the fields $F_{i}=k\left(a, \delta(a), \ldots, \delta^{i-1}(a)\right)$. For each $i \geq 1$, if $F_{i-1} \subsetneq F_{i}$, then since $\left[\delta^{i-1}(a)\right]^{p} \in k \subseteq F_{i-1}$, we must have $\left[F_{i}: F_{i-1}\right]=p$ and $F_{i-1} \subseteq F_{i}$ is a purely inseparable simple extension.

Suppose that $F_{i-1} \subsetneq F_{i}$ for all $i \geq 1$, let $F=\bigcup_{i \geq 0} F_{i}$, and note that $F$ is closed under $\delta$; so it is enough to prove that $F(x ; \delta)$ contains a free subalgebra. Write $b_{i}=\delta^{i}(a)$. By the analysis of the previous paragraph, it easily follows that $F$ has a $k$-basis consisting of all words in the $b_{i}$ of the form

$$
\left\{b_{0}^{e_{1}} b_{1}^{e_{2}} \ldots b_{m}^{e_{m}} \mid 0 \leq e_{j} \leq p-1\right\}
$$

Now we apply the criterion of Theorem 2.2, with the choice $b=b_{0}=a$. Thus it is sufficient to prove the claim that if $u \in F$ satisfies $\delta(u) \in k+k b$, then $u \in k$. To obtain this claim, suppose that $u$ satisfies $\delta(u) \in k+k b$ with $u \notin k$, so there exists some $d \geq 0$ such that $u \in F_{d+1} \backslash F_{d}$. We can write $u$ as

$$
u=\sum_{i=0}^{p-1} u_{i} b_{d}^{i}
$$


where each $u_{i} \in F_{d}$ and $u_{i} \neq 0$ for some $i>0$. Thus we see that

$$
\delta(u)=\sum_{i=0}^{p-1} i u_{i} b_{d}^{i-1} b_{d+1}+\sum_{i=0}^{p-1} \delta\left(u_{i}\right) b_{d}^{i},
$$

where the second sum is contained in $F_{d+1}$. Since some $u_{i} \neq 0$ with $i \neq 0$, we have $\delta(u) \notin F_{d+1}$, contradicting the assumption $\delta(u) \in k+k b$. This proves the claim, and so $K(x ; \delta)$ contains a free algebra on two generators.

On the other hand, suppose that for some $a \in K$, the sequence of fields

$$
F_{i}=k\left(a, \delta(a), \ldots, \delta^{i-1}(a)\right)
$$

has $F_{i}=F_{i+1}$ for some $i$. Then it is easy to see that $F_{n}=F_{i}$ for all $n \geq i$, and so $F_{i}$ is a $\delta$-invariant subfield, of finite degree over $k$. If this happens for every $a \in K$, then every finite subset of $K$ is contained in a $\delta$-invariant subfield $F$ of finite degree over $k$, and so is contained in the PI division ring $F(x ; \delta)$. Thus $K(x ; \delta)$ is locally PI and does not contain a noncommutative free subalgebra.

Proof of Theorem 1.2. Examining the proofs of parts (1) and (2) of Theorem 4.1, we see that $K(x ; \delta)$ contains a free subalgebra if and only if it is not locally PI.

An interesting example of part (2) of Theorem 4.1 is obtained by taking $K=$ $\mathbb{F}_{p}\left(x_{0}, x_{1}, \ldots\right)$ to be a rational function field in infinitely many indeterminates over the field of $p$ elements, and defining $\delta\left(x_{i}\right)=x_{i+1}$ for all $i \geq 0$. The ring $K(x ; \delta)$ then contains a free algebra in two generators over $\mathbb{F}_{p}$. This ring has appeared before in the literature and has other interesting properties. In particular, Resco and Small [1993] studied this ring as an example of a noetherian affine algebra which becomes non-noetherian after base field extension.

\section{Summary theorems}

In this section, we apply our results to show that the free subalgebra conjecture holds for a large class of algebras formed from iterated Ore extensions. We state our summary theorems over an uncountable field for convenience, though they hold over an arbitrary field whenever the iterated Ore extension is built out of extensions satisfying Theorem 1.1(2).

Before proving our main theorem, we make an easy observation. The reason that we have not yet considered Ore extensions with both an automorphism and derivation is the following fact.

Lemma 5.1. Let $D$ be a PI division algebra with automorphism $\sigma$ and $\sigma$-derivation $\delta$. Then $D[x ; \sigma, \delta]$ is isomorphic either to $D\left[x^{\prime} ; \sigma^{\prime}\right]$ for some other automorphism $\sigma^{\prime}$, or else to $D\left[x^{\prime} ; \delta^{\prime}\right]$ for some derivation $\delta^{\prime}$. 
Proof. This is presumably well-known, but we sketch the proof since it is elementary. Let $Z=Z(D)$. For any $a \in D, b \in Z$, we have $\delta(a b)=\delta(b a)$, and so

$$
\sigma(a) \delta(b)+\delta(a) b=\sigma(b) \delta(a)+\delta(b) a .
$$

Since $\sigma(b) \in Z$ also, we have $\delta(a)[b-\sigma(b)]=\delta(b) a-\sigma(a) \delta(b)$. Then if there is any $b \in Z$ such that $\sigma(b) \neq b$, we must have $\delta(a)=(b-\sigma(b))^{-1}[\delta(b) a-\sigma(a) \delta(b)]$ for all $a \in D$. In this case, making the change of variable $x^{\prime}=x+(b-\sigma(b))^{-1} \delta(b)$, one easily checks that $D[x ; \sigma, \delta] \cong D\left[x^{\prime} ; \sigma, 0\right]$.

Otherwise, $\sigma(b)=b$ for all $b \in Z$; in other words, $\sigma$ is trivial on the center. By the Skolem-Noether theorem, $\sigma$ is an inner automorphism of $D$, say $\sigma(a)=d^{-1} a d$ for all $a$ and some $d \in D^{\times}$. Then the change of variable $x^{\prime}=d x$ gives

$$
D[x ; \sigma, \delta] \cong D\left[x^{\prime} ; 1, d \delta\right] .
$$

Theorem 5.2. Let $k$ be an uncountable field. The following results hold:

(1) Let $A$ be any PI domain which is a k-algebra with automorphism $\sigma$ and $\sigma$ derivation $\delta$ (over $k$ ). Then the quotient division algebra of $A[x ; \sigma, \delta]$ satisfies the free subalgebra conjecture.

(2) If $A$ is any affine $k$-algebra which is an Ore domain such that $Q(A)$ satisfies the free subalgebra conjecture, then $Q(A[x ; \sigma, \delta])$ also satisfies the conjecture.

Proof. (1) Let $D$ be the quotient division algebra of $A$, so that $R$ has quotient ring $Q(R)=D(x ; \sigma, \delta)$. By Lemma 5.1, it is enough to consider the two special cases $D(x ; \sigma)$ and $D(x ; \delta)$. If $K=Z(D)$, then $\sigma$ restricts to $K$ and $D$ is finite over $K$ since $D$ is PI. It is easy to see that it is enough to prove the free subalgebra conjecture for $K(x ; \sigma)$. Now Theorem 1.1 gives the result.

Similarly, considering $D(x ; \delta)$, we have $\delta(K) \subseteq K$ (use (5.7)), and so we easily reduce to the case of $K(x ; \delta)$. We are done by Theorem 1.2.

(2) If $A$ is not locally PI, then by assumption, $Q(A)$ contains a free subalgebra on two generators. Then there is an embedding $Q(A) \subseteq Q(A[x ; \sigma, \delta])$, and of course $Q(A[x ; \sigma, \delta])$ is also not locally PI, so we are done in this case. If instead $A$ is locally PI, then it is actually PI by the assumption that $A$ is affine. Now part (1) applies.

Proof of Theorem 1.3. An easy induction using parts (1) and (2) of Theorem 5.2 shows that any iterated Ore extension of an affine PI domain over an uncountable field has a quotient division algebra satisfying the free subalgebra conjecture.

\section{Acknowledgments}

We thank James Zhang, Sue Sierra, Agata Smoktunowicz, Toby Stafford, and Lance Small for many valuable discussions. We thank Jairo Gonçalves for pointing out an 
error in a previous version of the paper. Finally, we are grateful to the referee for providing the proof of Proposition 3.2, for suggesting Remark 3.5, and for many helpful comments which improved the exposition of the paper.

\section{References}

[Bell and Rogalski 2011] J. P. Bell and D. Rogalski, "Free subalgebras of division algebras over uncountable fields", preprint, 2011. arXiv 1112.0041

[Bell et al. 2010] J. Bell, D. Rogalski, and S. J. Sierra, “The Dixmier-Moeglin equivalence for twisted homogeneous coordinate rings", Israel J. Math. 180 (2010), 461-507. MR 2011m:14005 Zbl 1219.16030

[Chiba 1996] K. Chiba, "Free subgroups and free subsemigroups of division rings", J. Algebra 184:2 (1996), 570-574. MR 97g:16030 Zbl 0865.16014

[Figueiredo et al. 1996] L. M. V. Figueiredo, J. Z. Gonçalves, and M. Shirvani, "Free group algebras in certain division rings", J. Algebra 185:2 (1996), 298-313. MR 97j:16030 Zbl 0866.16011

[Goncalves and Shirvani 1996] J. Goncalves and M. Shirvani, "On free group algebras in division rings with uncountable center", Proc. Amer. Math. Soc. 124:3 (1996), 685-687. MR 96f:16020 Zbl 0844.16012

[Hartshorne 1977] R. Hartshorne, Algebraic geometry, Graduate Texts in Mathematics 52, Springer, New York, 1977. MR 57 \#3116 Zbl 0367.14001

[Krause and Lenagan 2000] G. R. Krause and T. H. Lenagan, Growth of algebras and GelfandKirillov dimension, 2nd ed., Graduate Studies in Mathematics 22, American Mathematical Society, Providence, RI, 2000. MR 2000j:16035 Zbl 0957.16001

[Lichtman 1999] A. I. Lichtman, "Free subalgebras in division rings generated by universal enveloping algebras”, Algebra Colloq. 6:2 (1999), 145-153. MR 2001k:16030 Zbl 0971.16010

[Lorenz 1986] M. Lorenz, "On free subalgebras of certain division algebras", Proc. Amer. Math. Soc. 98:3 (1986), 401-405. MR 87m:16040 Zbl 0615.16002

[Makar-Limanov 1983] L. Makar-Limanov, "The skew field of fractions of the Weyl algebra contains a free noncommutative subalgebra”, Comm. Algebra 11:17 (1983), 2003-2006. MR 84j:16012 Zbl 0521.16015

[Makar-Limanov 1984a] L. Makar-Limanov, "On free subobjects of skew fields", pp. 281-285 in Methods in ring theory (Antwerp, 1983), NATO Adv. Sci. Inst. Ser. C Math. Phys. Sci. 129, Reidel, Dordrecht, 1984. MR 770598 Zbl 0539.16015

[Makar-Limanov 1984b] L. Makar-Limanov, "On free subsemigroups of skew fields", Proc. Amer. Math. Soc. 91:2 (1984), 189-191. MR 85j:16022 Zbl 0512.16018

[Makar-Limanov 1984c] L. Makar-Limanov, “On group rings of nilpotent groups”, Israel J. Math. 48:2-3 (1984), 244-248. MR 86c:16006 Zbl 0556.16006

[Makar-Limanov and Malcolmson 1991] L. Makar-Limanov and P. Malcolmson, "Free subalgebras of enveloping fields", Proc. Amer. Math. Soc. 111:2 (1991), 315-322. MR 91f:16023 Zbl 0719. 16008

[Reichstein and Vonessen 1995] Z. Reichstein and N. Vonessen, "Free subgroups of division algebras", Comm. Algebra 23:6 (1995), 2181-2185. MR 96a:16032 Zbl 0833.16015

[Resco and Small 1993] R. Resco and L. W. Small, "Affine Noetherian algebras and extensions of the base field”, Bull. London Math. Soc. 25:6 (1993), 549-552. MR 94m:16029 Zbl 0802.16014 
[Rogalski 2009] D. Rogalski, "GK-dimension of birationally commutative surfaces", Trans. Amer. Math. Soc. 361:11 (2009), 5921-5945. MR 2010j:14008 Zbl 1181.14005

[Shirvani and Gonçalves 1998] M. Shirvani and J. Z. Gonçalves, "Free group algebras in the field of fractions of differential polynomial rings and enveloping algebras", J. Algebra 204:2 (1998), 372-385. MR 99g:16033 Zbl 0932.16029

[Shirvani and Gonçalves 1999] M. Shirvani and J. Z. Gonçalves, "Large free algebras in the ring of fractions of skew polynomial rings", J. London Math. Soc. (2) 60:2 (1999), 481-489. MR 2000m: 16041 Zbl 0983.16023

Communicated by Susan Montgomery

Received 2011-03-10 Revised 2012-01-06 Accepted 2012-02-07

jpb@math.sfu.ca

Department of Mathematics, Simon Fraser University, 8888 University Drive, Burnaby, BC V5A 1S6, Canada http://www.math.sfu.ca/ jpb/

drogalsk@math.ucsd.edu

Department of Mathematics, University of California, San Diego, La Jolla, CA 92093-0112, United States http://www.math.ucsd.edu/ drogalsk/ 


\section{Algebra \& Number Theory}

msp.berkeley.edu/ant

\section{EDITORS}

MANAGING EDITOR

Bjorn Poonen

Massachusetts Institute of Technology

Cambridge, USA

\author{
EDITORIAL BOARD CHAIR \\ David Eisenbud \\ University of California \\ Berkeley, USA
}

\section{BOARD OF EDITORS}

Georgia Benkart

Dave Benson

Richard E. Borcherds

John H. Coates

J-L. Colliot-Thélène

Brian D. Conrad

Hélène Esnault

Hubert Flenner

Edward Frenkel

Andrew Granville

Joseph Gubeladze

Ehud Hrushovski

Craig Huneke

Mikhail Kapranov

Yujiro Kawamata

János Kollár

Yuri Manin

Barry Mazur

Philippe Michel
University of Wisconsin, Madison, USA

University of Aberdeen, Scotland

University of California, Berkeley, USA

University of Cambridge, UK

CNRS, Université Paris-Sud, France

University of Michigan, USA

Freie Universität Berlin, Germany

Ruhr-Universität, Germany

University of California, Berkeley, USA

Université de Montréal, Canada

San Francisco State University, USA

Hebrew University, Israel

University of Virginia, USA

Yale University, USA

University of Tokyo, Japan

Princeton University, USA

Northwestern University, USA

Harvard University, USA

École Polytechnique Fédérale de Lausanne
Susan Montgomery

Shigefumi Mori

Raman Parimala

Jonathan Pila

Victor Reiner

Karl Rubin

Peter Sarnak

Joseph H. Silverman

Michael Singer

Vasudevan Srinivas

J. Toby Stafford

Bernd Sturmfels

Richard Taylor

Ravi Vakil

Michel van den Bergh

Marie-France Vignéras

Kei-Ichi Watanabe

Andrei Zelevinsky

Efim Zelmanov
University of Southern California, USA

RIMS, Kyoto University, Japan

Emory University, USA

University of Oxford, UK

University of Minnesota, USA

University of California, Irvine, USA

Princeton University, USA

Brown University, USA

North Carolina State University, USA

Tata Inst. of Fund. Research, India

University of Michigan, USA

University of California, Berkeley, USA

Harvard University, USA

Stanford University, USA

Hasselt University, Belgium

Université Paris VII, France

Nihon University, Japan

Northeastern University, USA

University of California, San Diego, USA

\section{PRODUCTION}

production@msp.org

Silvio Levy, Scientific Editor

See inside back cover or www.jant.org for submission instructions.

The subscription price for 2012 is US \$175/year for the electronic version, and \$275/year ( $\$ 40$ shipping outside the US) for print and electronic. Subscriptions, requests for back issues from the last three years and changes of subscribers address should be sent to Mathematical Sciences Publishers, Department of Mathematics, University of California, Berkeley, CA 94720-3840, USA.

Algebra \& Number Theory (ISSN 1937-0652) at Mathematical Sciences Publishers, Department of Mathematics, University of California, Berkeley, CA 94720-3840 is published continuously online. Periodical rate postage paid at Berkeley, CA 94704, and additional mailing offices.

ANT peer review and production are managed by EditFLOW ${ }^{\circledR}$ from Mathematical Sciences Publishers.

PUBLISHED BY

mathematical sciences publishers

http://msp.org/

A NON-PROFIT CORPORATION

Typeset in IATEX

Copyright ( 2012 by Mathematical Sciences Publishers 


\section{Algebra \& Number Theory}

\section{Volume $6 \quad$ No. $7 \quad 2012$}

On the rank of the fibers of rational elliptic surfaces

Cecília Salgado

Néron's pairing and relative algebraic equivalence

CÉDRIC PÉPIN

Free subalgebras of quotient rings of Ore extensions

JASON P. BELL and DANIEL ROGALSKI

Classes de cycles motiviques étales

1369

BRUNO KAHN

Higher-order Maass forms

1409

ROELOF BRUGGEMAN and NiKOLAOS DIAMANTIS

Log canonical thresholds, $F$-pure thresholds, and nonstandard extensions

Bhargav Bhatt, Daniel J. Hernández, Lance Edward Miller and Mircea MUSTAȚĂ

The semistable reduction problem for the space of morphisms on $\mathbb{P}^{n}$

ALON LEVY

Grothendieck's trace map for arithmetic surfaces via residues and higher adèles

MATTHEW MORROW

Crystalline extensions and the weight part of Serre's conjecture

Toby GeE, Tong LiU and DAVID SAVITT

Annihilating the cohomology of group schemes 